\title{
ZUM STAND DER QUALITÄTSSICHERUNG NUKLEARMEDIZINISCHER MESSGERÄTE IN DER SCHWEIZ
}

\author{
H.W. Nemec, H.W. Roser \\ Abteilung Radiologische Physik, Kantonsspital/Universiätskliniken, CH-4031 Basel
}

\section{Zusammenfassung}

Seit dem 1. 1. 1998 ist in der Schweiz die "Verordnung über den Umgang mit offenen radioaktiven Strahlenquellen" in Kraft. Darin sind unter anderem Qualitätsprüfungen für nuklearmedizinische Mess- und Untersuchungsgeräte vorgeschrieben. Die Abnahmeprüfung für bildgebende Geräte ist durch den Hersteller nach NEMA, IEC oder äquivalenten Methoden durchzuführen. Für die Konstanzprüfung von in-vivo- und in-vitro-Messplätzen, Aktivimetern und Gammakameras werden die Prüfparameter und die Periodizität angegeben. Bei der Zustandsprüfung sind die Herstellerspezifikationen zu verifizieren.

Auf dem Hintergrund der neuen Verordnung werden die Erfahrungen mit Konstanzprüfungen, welche seit etwa 15 Jahren am Kantonsspital Basel durchgefüht werden sowie die Ergebnisse einer schweizerischen Erhebung vorgestellt. Abschliessend wird eine Methode beschrieben, welche die gleichzeitige Überprüfung der integralen Uniformität und der Langzeitkonstanz der Systemempfindlichkeit von Gammakameras gestattet, sowie auf die Bedeutung von Ringversuchen für die Prüfung von Aktivimetern hingewiesen.

\section{Einleitung}

Durch die Qualitätssicherung nuklearmedizinischer Messgeräte soll ein Höchstmass an diagnostischer Treffsicherheit bei einem Mindestmass an Strahlenexposition für Patienten, Personal und Umwelt erreicht werden [5]. Aus diesem Grund werden analog zu den entsprechenden Kontrollen in der Röntgendiagnostik seit etwa 15 Jahren am Kantonsspital Basel physikalische Qualitätskontrollen von Aktivitätsmessgeräten und von Gammakameras durchgeführt. Gemäss einer Umfrage wurden in den nuklearmedizinischen Instituten der Schweiz in den Jahren 1993/94 derartige Qualitätskontrollen nur zum Teil durchgeführt. In der seit dem 1. 1. 1998 gültigen "Verordnung über den Umgang mit offenen radioaktiven Stoffen" [7] (nachstehend "Verordnung" genannt) sind unter anderem die Mindestanforderungen für die Qualitätssicherung nuklearmedizinischer Mess- und Untersuchungsgeräte festgelegt Die wichtigsten Schlussfolgerungen daraus werden im folgenden beschrieben und in Beziehung zu früheren Ergebnissen gesetzt.

\section{Methoden}

Für die Qualitätskontrolle von Aktivimetern müssen geeignete Kalibrierquellen (Prüfstrahler) zur Verfügung stehen (z.B. Cs-137, Ba-133, Co-57). $\mathrm{Zu}$ jedem
Aktivimeter für die Messung von Tc-99m muss zudem eine Vorrichtung für die Prüfung auf MolybdänDurchbruch verfügbar sein [7].

Bei der Qualitätskontrolle von Gammakameras erfolgt die Überprüfung von Homogenität und Ausbeute mit entsprechenden Strahlenquellen, d.h. entweder mit Punktquellen (z.B. Tc-99m in Fläschchen oder Spritzen), Co-57-Flächenquellen oder mit einem Flächenphantom, welches mit radioaktiver Nuklidlösung (z.B. Tc-99m) gefüllt ist. Zur Überprüfung der geometrischen Auflösung und Linearität werden meist Bleistreifen- oder Lochphantome (z.B. BRH-Phantom) verwendet Bei SPECT-Kameras kommt zusätzlich ein spezielles Tomographie-Phantom zum Einsatz, welches verschiedenartige Inhomogenitäten enthält. Ausserdem ist eine Einrichtung zur Kontrolle des Rotationszentrums erforderlich.

\section{Ergebnisse}

Kantonsspital Basel seit 1983

Am Kantonsspital Basel werden seit etwa 15 Jahren Qualitätskontrollen an Gammakameras durchgeführt [3]. Diese Kontrollen erfolgten bis vor kurzem mit wenigen Ausnahmen in monatlichem Rhythmus und umfassten im wesentlichen relativ einfache Tests, welche ausgehend von verschiedenen internationalen Empfehlungen und Normen weiterentwickelt wurden. Dazu gehören insbesondere die Kontrolle der Energie, der Uniformität, des Photomultiplier-Abgleichs, der räumlichen Auflösung, der geometrischen Linearität, der zeitlichen Auflösung und der Untergrundzählrate. Neben einer mehr oder weniger subjektiven Beurteilung von Kontrollaufnahmen wurden die Testergebnisse zur Objektivierung der Beurteilung zum Teil mit Hilfe selbsterstellter Computerprogramme quantifiziert. Bei SPECT-Kameras wurden zusätzlich die Rotationsachse, die Qualität der tomographischen Aufnahme und gegebenenfalls der korrekte Ablauf im Ganzkörpermodus überprüft. Insgesamt wurden in 5 bis $10 \%$ der Kontrollen Mängel festgestellt. Diese betrafen in erster Linie die Homogenität bei den SPECTKameras, den Zustand der Photomultiplier, die geometrische Linearität und die geometrische Auflösung.

Ausserdem werden seit 1983 am Kantonsspital Basel physikalische Qualitätskontrollen von Aktivitätsmessgeräten (Aktivimetern) durchgeführt [2]. Diese Kontrollen umfassen die Messgenauigkeit und die Reproduzierbarkeit für kalibrierte Gammastrahler unter Standardgeometrie, die Linearität der Anzeige in Abhängigkeit von der Aktivität, den Nulleffekt unter 
empfindlichsten Messbedingungen, den Mo-99Durchbruch bei der Messung von Tc-99m, die Korrekturfaktoren für nicht-standardmässige Messgeometrie, die Reproduzierbarkeit (zeitliche Stabilität, gemessen mit langlebigen umschlossenen Gammastrahlern) und den Nulleffekt unter betriebsmässigen Messbedingungen. Diese Routinekontrollen wurden bisher mindestens einmal monatlich sowie nach jeder Reparatur bzw. Wartung durchgefuihrt.

\section{Schweizerische Erhebung 1993/94}

In den Jahren 1993/94 wurde vom Institut für Nuklearmedizin sowie von der Abteilung Radiologische Physik des Kantonsspitals Basel eine Erhebung an insgesamt 21 nuklearmedizinischen Instituten der Schweiz durchgeführ. Diese Anzahl entspricht etwa der Hälfte aller Schweizer Institutionen, in welchen nuklearmedizinische Diagnostik betrieben wird, wobei insbesondere alle Universitätskliniken und grösseren Spitäler eingeschlossen sind. Dabei wurden unter anderem Informationen über Art und Häufigkeit von Qualitätskontrollen an Gammakameras eingeholt. Mehrheitlich wurde die Kontrolle der Uniformität - in der Regel mit einer Co-57-Flächenquelle - sowie teilweise auch der Energie und der Empfindlichkeit genannt. Die Häufigkeit variierte von täglich bis monatlich. Von einigen Anwendern wurden als weitere Kontrollen, welche wöchentlich bis monatlich durchgefuihrt wurden, angegeben: Linearität, Auflösung, maximale Zählrate sowie bei SPECT-Kameras das Rotationszentrum.

\section{"Verordnung" seit 1.1.1998}

Die seit kurzem gültige "Verordnung" [7] enthält unter anderem Vorschriften für die medizinischen Anwendungen radioaktiver Stoffe. Bei den Qualitätsprüfungen für nuklearmedizinische Mess- und Untersuchungsgeräte werden Abnahme, Konstanz- und Zustandsprüfungen unterschieden. Im Rahmen der Abnahmeprüfung hat der Lieferant eines Gerätes die Herstellerspezifikationen gemäss NEMA, IEC oder äquivalenten Methoden zu verifizieren. Die Konstanzprüfungen sind für Aktivimeter in Tabelle 1 und für Gammakameras in Tabelle 2 aufgeführt.

\section{Tabelle 1: Konstanzprüfungen für Aktivimeter gemäss "Verordnung" [7]}

\begin{tabular}{|l|l|}
\hline Prüfparameter & Periodizität \\
\hline -Untergrundzählrate (Nulleffekt) und & täglich \\
Ausbeute in einer Nuklideinstellung & \\
-Untergrundzählrate (Nulleffekt) und & wöchentlich \\
Ausbeute in allen benutzten Nuklid- & \\
einstellungen & \\
-Untergrundzählrate (Nulleffekt), & halbjährlich \\
Ausbeute und Linearität in allen & \\
Nuklideinstellungen mit geeigneter & \\
Prüfquelle & \\
\hline
\end{tabular}

Zustandprüfungen sind mindestens jährlich sowie nach Wartungen und Reparaturen durchzuruihren. Dabei sind jeweils, analog zur Abnahmeprüfung, die Herstellerspezifikationen nach NEMA und IEC zu verifizieren.
Es kann auch genügen, ausgewählte Parameter zu überprüfen. Dazu gehören mindestens Homogenität, Energieauflösung, intrinsische Auflösung, Systemauflösung, Systemempfindlichkeit (Ausbeute) und Zählratenverhalten, Abbildungsmasstab und Dokumentationseinrichtung.

Tabelle 2: Konstanzprüfungen für Gammakameras gemäss "Verordnung" [7]

\begin{tabular}{|l|l|}
\hline Prüfparameter & Periodizität \\
\hline -Untergrundzählrate (Nulleffekt), & täglich \\
Einstellung des Energiefensters & \\
-Homogenität, Ausbeute & wöchentlich \\
-geometrische Linearität, Auflösung, & halbjährlich \\
Ganzkörper-Zusatz; bei Kameras zu & \\
tomographischen Untersuchungen & \\
zudem die Korrekturwerte für das & \\
Rotationszentrum und die Qualität & \\
der Schnittbilder & \\
\hline
\end{tabular}

\section{Prüfung von Uniformität und Ausbeute}

Gemäss einer kürzlich beschriebenen Methode [4] kann die Inhomogenität von Gammakameras als Systeminhomogenitä̆, das heisst bei aufgesetztem Kollimator, mit einer Flächenquelle überprüft werden. Trotz der gegenüber Tc-99m unterschiedlichen Energie wird aufgrund der einfacheren Handhabung eine Co-57Festkörperquelle verwendet. Die Messzeit beträgt 15 Minuten, die Impulszahl liegt zwischen $1 \times 10^{7}$ und $4 \times 10^{7}$. Bei einer Ausgangsaktivität von $740 \mathrm{MBq}$ liefert die Flächenquelle während etwa 1,5 Jahren eine ausreichende Zählstatistik. Der Anteil der Zählrate des Untergrundes an der gesamten Zählrate bleibt bis zum Quellenwechsel unterhalb von $5 \times 10^{-3}$.

Aufgrund der Messergebnisse lässt sich bei gleichbleibender Positionierung der Quelle unter Berücksichtigung der physikalischen Halbwertszeit von Co-57 (272 Tage) die Flutfeldaufnahme, welche zur Überprüfung der integralen Uniformität über das UFOV (useful field of view) gemessen wird, auch zur Kontrolle der Langzeitkonstanz der Systemempfindlichkeit (Ausbeute) verwenden.

\section{Jährlicher Ringversuch für Aktivimeter}

Gemäss "Verordnung" [7] sind Aktivimeter mindestens jährlich einer Eichung oder einer Vergleichsmessung durch das Eidgenössische Amt für Messwesen (EAM) oder durch eine von ihm anerkannte Stelle zu unterziehen. Derartige Ringversuche mit jährlich wechselnden Radionuklidlösungen werden bereits seit mehreren Jahren durch das vom EAM beauftragte "Institut de Radiophysique Appliquée" in Lausanne durchgefuihrt. Im Jahre 1997 nahmen 38 nuklearmedizinische Institute - und damit die Mehrzahl von Radionuklid-Anwendern in der Medizin - an der Vergleichsmessung mit Tl-201 teil. Abgesehen von zwei extremen Abweichungen ergaben die meisten der von 6 verschiedenen Herstellerm gelieferten Aktivimeter Werte, welche innerhalb des von der Europäischen Pharmakopöe geforderten Normbereichs von $+/-10 \%$ lagen [1].

\section{Biomedizinische Technik Band 43 Ergänzungsband 21998}




\section{Diskussion}

Die effektive Dosis für eine einzelne nuklearmedizinische Untersuchung beträgt für einen Patienten im Mittel $4 \mathrm{mSv}$ und liegt damit in der Grössenordnung der mittleren jährlichen Exposition in der Schweiz. Je nach Untersuchung können die effektiven Dosen jedoch bis zu $30 \mathrm{mSv}$ und mehr betragen [6]. $\mathrm{Zu}$ den Möglichkeiten der Dosisreduzierung gehört auch die Qualitätssicherung der eingesetzen Apparaturen. Die Notwendigkeit von Qualitätskontrollen zeigen auch die Ergebnisse eigener Untersuchungen über Art und Häufigkeit von Mängeln an Gammakameras [3]. Wie die Umfrage von 1993/94 zeigt, wurden jedoch ohne gesetzliche Vorschriften derartige Massnahmen nur zum Teil durchgeführt.

Eine weitere Bedingung für die Umsetzung der neuen Verordnung besteht in der einfachen Durchührbarkeit der Kontrollen bei möglichst geringem Zeitaufwand. Diesem Zweck dient die oben erwähnte Überprüfung von Homogenität und Ausbeute in einem einzigen Arbeitsgang [4]. Ausserdem wird zur Sicherstellung der exakten Aktivitätsmenge jährlich ein von der "Verordnung" [7] vorgeschriebener Ringversuch zur Kalibrierung der Aktivimeter mit geeigneten Prüfquellen durchgefuihrt [1]. Deshalb sollte aufgrund der seit Beginn dieses Jahres geltenden "Verordnung" [7] in der Schweiz der Zustand der nuklearmedizinischen Mess- und Untersuchungsgeräte verbessert werden, wobei die Verordnung unseres Erachtens nur das absolute Minimum an notwendigen Massnahmen verlangt.

\section{Literatur}

[1] J.-J. Gostely, M. Decombaz: Campagne d' étalonnage ${ }^{201}$ TV1997. Institut de Radiophysique Appliquée, Lausanne 1997

[2] H.W. Nemec: Zur Bedeutung physikalischer Qualitätskontrollen von Aktivitätsmessgeräten. In: Neue Aspekte radiologischer Diagnostik und Therapie. Jahrbuch 1984 der SGRNM (Red. W. Bessler et al.), S. 286-291, Verlag Hans Huber, Bern-Stuttgart-Toronto 1985

[3] H.W. Nemec, H.-P. Hafner, J. Roth: Qualitätskontrollen von Gamma-Kameras: Erfahrungen mit einfachen Tests. In: Neue Aspekte radiologischer Diagnostik und Therapie. Jahrbuch 1986 der SGRNM (Red. W. Bessler et al.), S. 147-153, Verlag Hans Huber, Bern-Stuttgart-Toronto 1987

[4] H.W. Nemec, H.W. Roser: Zur Konstanzprüfung der Systemempfindlichkeit (Ausbeute) von Gammakameras. Medizinische Physik 1997, Jahrestagung der DGMP (Hrsg. R. Schmidt), S. 273-274, Hamburg 1997 [5] H.D. Roedler: Qualitätskontrollen nuklearmedizinischer Messgeräte. Z. Med. Phys. 3 (1993) 110-115

[6] H.W. Roser, J. Roth: Die Strahlenexposition infolge nuklearmedizinischer Untersuchungen in der Schweiz. Z. Med. Phys. 8 (1998) 41-48

[7] Verordnung über den Umgang mit offenen radioaktiven Strahlenquellen (vom 21 . November 1997). EDMZ, Bern 1998

\section{Adresse}

H.W. Nemec, H.W. Roser, Abteilung Radiologische Physik, Kantonsspital/Universitätskliniken, $\mathrm{CH}-4031$ Basel, Tel. $(++41)+61.2653143$, Fax: $(++41)+61.2653135$, E-Mail: nemec@ubaclu.unibas.ch 